\title{
Erratum to: Numerical integration over the square in the presence of algebraic/logarithmic singularities with an application to aerodynamics
}

\section{Walter Gautschi}

Published online: 6 November 2013

(C) Springer Science+Business Media New York 2013

\section{Erratum to: Numer Algor (2012) 61:275-290}

\section{DOI 10.1007/s11075-012-9611-9}

Unfortunately, there is a misprint in Eq. (2.7): at the end of the second line of the equation, $1-x_{\mu}$ should read $1-\xi_{\mu}$. The Matlab program Ial.m using this equation is not affected by this misprint. The author is indebted to Luc Paquet for bringing this error to his attention. The corrected version of equation is given below.

$$
\begin{array}{r}
I_{\log } \approx-\sum_{\mu=1}^{n} \sum_{v=1}^{n}\left\{B_{\mu v}\left[f\left(\left(1-\xi_{v}\right) x_{\mu}, x_{\mu}\right)+f\left(1-\left(1-\xi_{v}\right) x_{\mu}, 1-x_{\mu}\right)\right]\right. \\
\left.+C_{\mu v}\left[f\left(\left(1-x_{v}\right) \xi_{\mu}, \xi_{\mu}\right)+f\left(1-\left(1-x_{v}\right) \xi_{\mu}, 1-\xi_{\mu}\right)\right]\right\},
\end{array}
$$

The online version of the original article can be found at http://dx.doi.org/10.1007/s11075-012-9611-9.

W. Gautschi $(\bowtie)$

Department of Computer Sciences, Purdue University, West Lafayette, IN 47907-2066, USA

e-mail:wgautschi@purdue.edu 